\title{
The hierarchical hub median problem with single assignment
}

\author{
Hande Yaman \\ Bilkent University, Department of Industrial Engineering, Bilkent, 06800 Ankara, Turkey
}

\section{A R T I C L E I N F O}

\section{Article history:}

Received 29 July 2008

Received in revised form 27 January 2009

Accepted 27 January 2009

\section{Keywords:}

Hub location

p-Hub median

Hierarchical

Time restrictions

\begin{abstract}
A B S T R A C T
We study the problem of designing a three level hub network where the top level consists of a complete network connecting the so-called central hubs and the second and third levels are unions of star networks connecting the remaining hubs to central hubs and the demand centers to hubs and central hubs, respectively. The problem is to decide on the locations of a predetermined number of hubs and central hubs and the connections in order to minimize the total routing cost in the resulting network. This problem includes the classical $p$-hub median problem as a special case. We also consider a version of this problem where service quality considerations are incorporated through delivery time restrictions. We propose mixed integer programming models for these two problems and report the outcomes of a computational study using the CAB data and the Turkey data.
\end{abstract} (C) 2009 Elsevier Ltd. All rights reserved.

\section{Introduction}

Given a set of nodes, the traffic demand and the unit transportation cost between pairs of nodes, we are interested in the problem of constructing a network to route the traffic at minimum cost. One possible solution is to connect all pair of nodes by direct links. However, the amount of traffic demand between a pair of nodes may not always justify economically the allocation of a transportation mode on this link. The idea of hubbing is to consolidate traffic to profit from the economies of scale. Hence hubs are located at some of the nodes of the network and serve as consolidating points.

In a classical hub network, the hubs are connected with a complete network. Each remaining node is connected to hubs by direct links and all the traffic that this node wants to send and receive travels on these links. Here we consider the single assignment version where each remaining node is connected to exactly one hub with direct links. Hence, the network formed by a hub and the nodes assigned to it forms a star network. As a result, the classical single assignment hub network has two levels, the top level being a complete network connecting the hub nodes and the second level being a set of star networks each connecting a hub and the nodes assigned to it. The traffic originating at a node travels from the node to its hub on the direct link between the node and the hub, then it travels from this hub to the hub of the destination node on the direct link between these two hubs and finally from the hub of the destination node to the destination node on the direct link connecting these two nodes. If the origin and the destination are served by the same hubs, then the traffic travels from the origin to the hub and then from the hub to its destination. Hence the traffic between two nodes travels on at most three links traversing at most two hubs. Usually, the routing cost between two hub nodes is discounted at a rate of $\alpha$ to reflect the savings due to economies of scale. The $p$-hub median problem with single assignment is the problem of selecting $p$ hubs and assigning each demand node to exactly one hub to minimize the cost of routing the traffic. This problem has applications in transportation and telecommunications. 
The research on the $p$-hub median problem with single assignment started with the works of O'Kelly (1986, 1987). Campbell (1994), Ebery (2001), Ernst and Krishnamoorthy (1996), O’Kelly et al. (1996), Skorin-Kapov et al. (1996), and Sohn and Park (1997, 1998) present formulations for the problem. Various heuristic algorithms have been developed by AbdinnourHelm (2001), Campbell (1996), Ernst and Krishnamoorthy (1996), Klincewicz (1991, 1992), O'Kelly (1987), Pirkul and Schilling (1998), Skorin-Kapov and Skorin-Kapov (1994), and Smith et al. (1996). Ernst and Krishnamoorthy (1998) propose a branch and bound method where shortest path problems are solved to compute lower bounds. For more details, we refer the readers to two recent surveys, Campbell et al. (2002) and Alumur and Kara (2008).

One of the basic assumptions of the $p$-hub median problem with single assignment is that direct connections between all pairs of hubs can be justified economically. In this paper, we relax this assumption by introducing two types of hubs and a three level network where the top level connects central hubs with a complete network, the second level connects remaining hubs to the central hubs through star networks and the third level connects the demand nodes to the hubs with star networks. The study of this hierarchical structure is motivated by an application in cargo delivery. The investigation of a major cargo company in Turkey (see Elmastas, 2006) showed that the company uses such a three level structure where two central hubs located in Ankara and Istanbul are connected with direct links, each remaining hub is connected to one of these two central hubs and each remaining demand node is connected to a hub or a central hub. In this network, all traffic originating at a demand node first travels to the hub to which this node is assigned. The traffic with destination nodes that are assigned to the same hub are separated to be sent directly to the destination. The remaining traffic is combined with the traffic from the other nodes that are assigned to this hub and is forwarded to the central hub to which the hub is connected. At the central hub, the traffic with destination nodes that are served by hubs that are assigned to this same central hub is separated. The remaining traffic is sent to the other central hubs according to the assignments of the destination nodes. A central hub that receives traffic from other central hubs, sorts the traffic, combines it with the traffic that it receives from the hubs and demand nodes that are assigned to it, and then sends the traffic on to the appropriate hubs and demand nodes. Then each hub forwards the traffic it receives from its central hub and its demand nodes to the destination nodes.

In this hierarchical network, the traffic from an origin to a destination may visit up to four hubs on its way. If the origin and destination are assigned to the same hub then the traffic follows the path origin-hub-destination. If they are assigned to two different hubs that are assigned to the same central hub, then the path is origin-hub of the origin-central hub-hub of the destination-destination. If the origin and destination are assigned to two different hubs that are assigned to two different central hubs, then the traffic follows the path origin-hub of the origin-central hub of the hub of the origin-central hub of the hub of the destination-hub of the destination-destination. A leg on a path may be vacuous if its end points correspond to the same nodes. For instance, if the hub of the origin is a central hub, then the leg (hub of the origin-central hub of the hub of the origin) is vacuous.

In Fig. 1, we have a network with 35 nodes where the first four nodes are central hubs and the first 11 nodes are hubs. The traffic from node 16 to node 20 follows the path $16 \rightarrow 6 \rightarrow 1 \rightarrow 3 \rightarrow 8 \rightarrow 20$ traversing four hubs in total, two of which are central hubs. The traffic from node 16 to node 12 follows the path $16 \rightarrow 6 \rightarrow 1 \rightarrow 5 \rightarrow 12$. This path visits three hubs of which only one is a central hub. Finally, the traffic from node 16 to node 15 goes through a single hub, namely hub 6 , taking the path $16 \rightarrow 6 \rightarrow 15$.

In a classical hub network, a path connecting an origin-destination pair visits at most two hubs. In a hierarchical hub network, it is possible to have paths visiting four hubs between origin-destination pairs. In the cargo delivery application, this requires multiple loading, unloading, sorting operations and a more involved coordination between demand points, hubs and central hubs.

The hierarchical structure discussed above also appears in the design of telecommunication networks. Wu et al. (1988) consider an intra-local access transport area network architecture. The demand nodes are the central offices and each central office is served by a hub. A group of central offices served by the same hub is called a cluster. Each hub is then connected to a gateway (central hub). A group of clusters served by the same gateway is called a sector. Gateways are connected by a complete fiber network. This network architecture is the same as the three level network described above.

In this paper, we investigate the following problem. Given a set of demand nodes, a set of possible locations for hubs, a set of possible locations for central hubs, the number of hubs and central hubs to be opened, the traffic demand and the routing cost between pairs of nodes, and the discount factors due to economies of scale, determine the locations of hubs and central hubs, the assignment of demand nodes to hubs and the assignment of hubs to central hubs to minimize the total routing cost in the network. We call this problem the Hierarchical Hub Median Problem with Single Assignment and abbreviate it with SA-HHM.

One of the important issues for cargo delivery companies is delivery times. Customer satisfaction is highly affected by delivery times and delivering in shorter times brings a competitive advantage to companies. Incorporating delivery times into hub location models has been studied in various papers (see e.g., Ernst et al., 2005; Kara and Tansel, 2001, 2003; Tan and Kara, 2007; Wagner, 2004, 2008; Yaman et al., 2007). In this paper, we also model the Time Restricted Hierarchical Hub Median Problem with Single Assignment ( $S A-T H-H M$ ) where we add to SA-H-HM the requirement that all cargo is delivered to destinations within a given time bound.

In telecommunications networks, connecting origin-destination pairs using paths with bounded lengths is important for the quality of service. The problem $S A-T H-H M$ incorporates this notion into the design problem by taking the traveling times equal to distances or transmission times. 


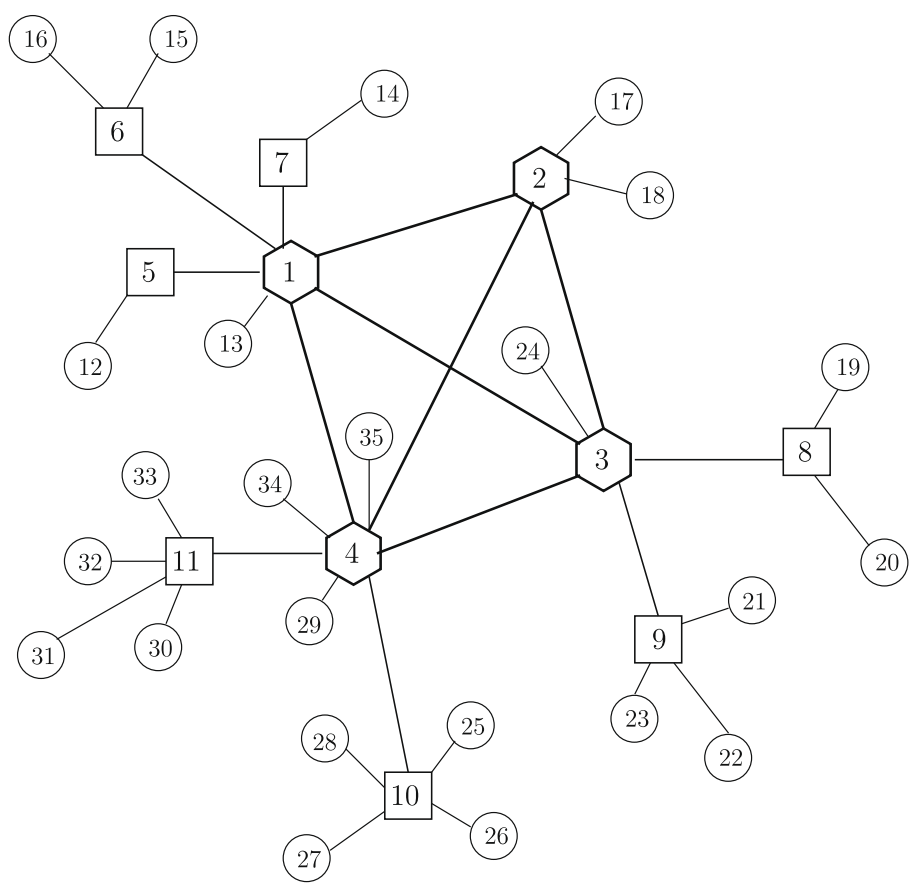

Fig. 1. A three level network on 35 nodes with 11 hubs and 4 central hubs.

To the best of our knowledge, there is no previous study on SA-H-HM and on SA-TH-HM. A three level network is considered in Elmastas (2006) where the design problem of a cargo delivery company which uses both airplanes and trucks is modeled and solved. There are two major differences with the problem of this paper. The network in Elmastas (2006) has a different structure: the top level connecting hub airports is a star, the second level that connects hubs among themselves and to hub airports has a mesh structure and the third level connecting demand points to hubs is composed of star networks. The second difference is the objective of the problem. The aim is to minimize the fixed cost of using planes and operating segments under the constraint of delivering all cargo demand within a given time bound whereas here we minimize the total routing cost subject to a time bound.

The contribution of the present paper is to define two new hub location problems, to propose models for them and to present computational results to evaluate our capabilities in solving the proposed models. Results obtained for the CAB data and the Turkey data are given with emphasis on the effects of the choice of parameters and the delivery time restrictions on the total cost and the locations of central hubs.

The rest of the paper is organized as follows: in Section 2, we present a nonlinear $0-1$ formulation for SA-H-HM. A mixed integer programming formulation is given in Section 3. In Section 4, we discuss how we incorporate delivery time restrictions to our hub location problem and present a model for SA-TH-HM. The outcomes of our computational study are discussed in Section 5. We conclude the paper in Section 6.

\section{A nonlinear 0-1 model for $S A-H-H M$}

In this section, we first give the notation and then present a nonlinear $0-1$ model for our problem.

Let $I$ be the set of nodes, $H \subseteq I$ be the set of possible locations for hubs, and $C \subseteq H$ be the set of possible locations for central hubs. Let $p$ denote the number of hubs and $p_{0}$ denote the number of central hubs to be opened.

Let $t_{i m}$ denote the amount of traffic to be routed from node $i \in I$ to node $m \in I$. As the traffic from a node to itself does not travel on the links of the network, we assume that $t_{i i}=0$ for all $i \in I$.

Let $d_{i j}$ be the cost of routing a unit traffic from node $i \in I$ to node $j \in I$. We also assume that $d_{i j}=d_{j i}$ for all pair of nodes $i$ and $j$ and $d_{i i}=0$ for all $i$. We denote by $\alpha_{H}$ and $\alpha_{C}$, the reduction in routing cost between hubs and central hubs and the reduction in routing cost among central hubs, respectively.

To model $S A-H-H M$, we define two types of assignment variables. The first level assignment variable $y_{j l}$ is 1 if hub $j \in H$ is assigned to central hub $l \in C$ and 0 otherwise and the second level assignment variable $x_{i j}$ is 1 if node $i \in I$ is assigned to hub node $j \in H$ and 0 otherwise. If node $j \in H$ is a hub then $x_{j j}$ takes value 1 and if node $l \in H$ is a central hub then both $x_{l l}$ and $y_{l l}$ take value 1 . With these two sets of variables, we can obtain a nonlinear formulation of the problem: 


$$
\begin{array}{ll}
\min & \sum_{i \in I} \sum_{m \in I} t_{i m}\left(\sum_{j \in H} d_{i j} x_{i j}+\sum_{j \in H} \sum_{l \in C} \alpha_{H} d_{j l} x_{i j}\left(1-x_{m j}\right) y_{j l}\right. \\
& \left.+\sum_{l \in C} \sum_{v \in C} \alpha_{C} d_{l v} \sum_{j \in H} x_{i j} y_{j l} \sum_{u \in H} x_{m u} y_{u v}+\sum_{u \in H} \sum_{v \in C} \alpha_{H} d_{v u} x_{m u}\left(1-x_{i u}\right) y_{u v}+\sum_{u \in H} d_{u m} x_{m u}\right) \\
\text { s.t. } \quad & \sum_{j \in H} x_{i j}=1 \quad \forall i \in I \\
& \sum_{j \in H} x_{j j}=p \\
& x_{i j} \leqslant x_{j j} \quad \forall i \in I, j \in H \\
& \sum_{l \in C} y_{j l}=x_{j j} \quad \forall j \in H \\
& \sum_{l \in C} y_{l l}=p_{0} \quad \forall \\
& y_{j l} \leqslant y_{l l} \quad \forall j \in H, l \in C \\
& x_{i j} \in\{0,1\} \quad \forall i \in I, j \in H \\
& y_{j l} \in\{0,1\} \quad \forall j \in H, l \in C .
\end{array}
$$

Constraints (2), (4) and (8) ensure that each node in set $I$ is assigned to exactly one hub node and constraints (5), (7) and (9) ensure that each hub node is assigned to exactly one central hub node. The number of hubs is $p$ due to constraint (3) and the number of central hubs is $p_{0}$ due to constraint (6). The objective function is the total cost of routing in the network. The traffic from node $i$ to node $m$ goes from node $i$ to hub $j$ incurring a cost of $d_{i j}$ if node $i$ is assigned to hub $j$ (i.e., if $x_{i j}=1$ ) and then goes from hub $j$ to central hub $l$ if node $i$ is assigned to hub $j$, node $m$ is assigned to another hub and hub $j$ is assigned to central hub $l$ (i.e., if $x_{i j}=1, x_{m j}=0$, and $y_{j l}=1$ ). Here the routing cost $d_{j l}$ is discounted at a factor of $\alpha_{H}$. Then this traffic goes from central hub $l$ to central hub $v$ if node $i$ is assigned to a hub that is assigned to central hub $l$ and node $m$ is assigned to a hub that is assigned to central hub $v$ (i.e., if $\sum_{j \in H} x_{i j} y_{j l}=1$ and $\sum_{u \in H} x_{m u} y_{u v}=1$ ). On this part of the trip, the routing cost $d_{l v}$ is discounted at a rate of $\alpha_{C}$. If node $m$ is assigned to hub $u$ that is assigned to central hub $v$ and if node $i$ is not assigned to hub $u$, then the traffic goes from node $v$ to node $u$ (i.e., if $x_{m u}=1, x_{i u}=0$, and $y_{u v}=1$ ) with a routing cost of $d_{v u}$ discounted at $\alpha_{H}$. Finally, the traffic travels from hub $u$ to node $m$ incurring a cost of $d_{u m}$ if node $m$ is assigned to hub $u$ (i.e., if $x_{m u}=1$ ).

Setting $p=p_{0}$ forces all hubs to be central hubs. Letting $\alpha_{H}=\alpha_{C}=\alpha$, we obtain the classical $p$-hub median problem as a special case of our problem. As the $p$-hub median problem is NP-hard, SA-H-HM is also NP-hard.

Setting $p_{0}=1$ leads us to a network where $p-1$ hubs are selected and connected to a root node. Then all three levels of the network have star structures. Variants of this problem where the root node is given a priori are studied in the literature (see e.g., Labbé and Yaman, 2008; Yaman, 2008).

A major difference of our nonlinear formulation from the one for the classical $p$-hub median problem with single assignment is that it involves terms that are multiplications of more than two variables. It is possible to obtain a linear version of the above model by defining a new variable for each nonlinear term. This leads to a large formulation which may be of very little practical use.

\section{A mixed integer programming formulation for $S A-H-H M$}

In this section, we propose a mixed integer programming model that involves 3-index variables. We first review the formulations for the classical $p$-hub median problem with single assignment. O'Kelly (1987) proposes a quadratic mixed 0-1 model. Campbell (1994) and Skorin-Kapov et al. (1996) present linear models where 4-index variables are used. Ernst and Krishnamoorthy (1996) model the traffic as multicommodity flows aggregated by origin thus using 3-index variables. A formulation with 2-index variables and exponentially many constraints for the hub location problem with fixed costs is given in Labbé et al. (2005). A 2-index formulation with polynomial number of constraints is proposed by Ebery (2001). The author reports that this last formulation is not computationally efficient in general.

Now we present our model for SA-H-HM. We define two level assignment variables to differentiate the flow between hubs and central hubs and the flow among central hubs. The variable $z_{i j l}$ is 1 if node $i \in I$ is assigned to hub $j \in H$ and hub $j$ is assigned to central hub $l \in C$ and is 0 otherwise. Let $g_{j l}^{i}$ denote the amount of traffic which has node $i \in I$ as origin or destination and which travels between hub $j \in H$ and central hub $l \in C$ and $f_{k l}^{i}$ denote the amount of traffic which has node $i \in I$ as origin and which travels from central hub $k \in C$ to central hub $l \in C \backslash\{k\}$.

To be able to compute the amount of traffic on the links for a given assignment, we use the ideas developed in Ernst and Krishnamoorthy (1996) and Ebery (2001). The model we propose below is valid for routing cost vectors that satisfy the triangle inequality, i.e., we require that $d_{i j}+d_{j k} \geqslant d_{i k}$ for all nodes $i, j, k$ in $I$. 
We propose the following model for $S A-H-H M$.

$$
\begin{array}{ll}
\min & \sum_{i \in I} \sum_{m \in I}\left(t_{i m}+t_{m i}\right) \sum_{j \in H} d_{i j} \sum_{l \in C} z_{i j l}+\sum_{i \in I} \sum_{j \in H} \sum_{l \in C \backslash\{j\}} \alpha_{H} d_{j l} g_{j l}^{i}+\sum_{i \in I} \sum_{j \in C} \sum_{l \in C \backslash\{j\}} \alpha_{C} d_{j j} f_{j l}^{i} \\
\text { s.t. } & \sum_{j \in H} \sum_{l \in C} z_{i j l}=1 \quad \forall i \in I \\
& z_{i j l} \leqslant z_{j j l} \quad \forall i \in I, j \in H \backslash\{i\}, l \in C \\
& \sum_{m \in H} z_{j m l} \leqslant z_{l l l} \quad \forall j \in H, l \in C \backslash\{j\} \\
& \sum_{j \in H} \sum_{l \in C} z_{j j l}=p \\
& \sum_{l \in C} z_{l l l}=p_{0} \\
& \sum_{k \in C\{l\}} f_{l k}^{i}-\sum_{k \in C \backslash\{l\}} f_{k l}^{i}=\sum_{m \in I} t_{i m} \sum_{j \in H}\left(z_{i j l}-z_{m j l}\right) \quad \forall i \in I, l \in C \\
& g_{j l}^{i} \geqslant \sum_{m \in I \backslash j\}}\left(t_{i m}+t_{m i}\right)\left(z_{i j l}-z_{m j l}\right) \quad \forall i \in I, j \in H, l \in C \backslash\{j\} \\
& z_{l j l}=0 \quad \forall j \in H, l \in C \backslash\{j\} \\
& g_{j l}^{i} \geqslant 0 \quad \forall i \in I, j \in H, l \in C \\
& f_{k l}^{i} \geqslant 0 \quad \forall i \in I, k \in C, l \in C \backslash\{k\} \\
& z_{i j l} \in\{0,1\} \quad \forall i \in I, j \in H, l \in C .
\end{array}
$$

Constraints (11) ensure that each demand node is assigned to a hub and a central hub. Constraints (12) state that if a node $i$ is assigned to hub $j$ and central hub $l$, then node $j$ should be a hub assigned to central hub $l$. Due to constraints (13), if node $j$ is assigned to central hub $l$, then $l$ must be a central hub. The number of hubs and central hubs to be opened is fixed to $p$ and $p_{0}$, respectively, with constraints (14) and (15).

If node $i$ assigned to a hub that is assigned to central hub $l$, then the traffic from node $i$ to the nodes that are assigned to other central hubs leaves node $l$. If node $i$ is not assigned to central hub $l$, then the traffic from node $i$ to the nodes that are assigned to central hub $l$ enters node $l$. These are ensured by flow balance constraints (16).

Constraints (17) and (19) compute $g_{j l}^{i}$ values in terms of the assignment variables. The traffic adjacent at node $i$ and traveling between hub node $j$ and central hub $l$ is the traffic between node $i$ and the nodes that are not assigned to hub $j$ if node $i$ is assigned to hub $j$ and central hub $l$. Otherwise this amount is zero. Note here that the traffic between nodes $i$ and $j$ does not travel from hub $j$ to central hub $l$ if $i$ is assigned to hub $j$. Excluding index $j$ from the summation is not necessary for the validity of the model since if $z_{i j l}=1$ then $z_{j j l}=1$. But this strengthens the linear programming (LP) relaxation as $z_{i j l} \leqslant z_{j j l}$.

Constraints (18) are redundant but they again strengthen the LP relaxation.

The objective function (10) is equal to the sum of the costs of routing traffic between demand nodes and their hubs, between the hubs and their central hubs, and among central hubs.

Before concluding this section, we note that capacity constraints for hubs and central hubs can be easily incorporated into this model.

Also as multiple handlings may arise due to the hierarchical structure of the network, loading/unloading costs per unit traffic may be added to unit routing costs between distinct pairs of nodes to discriminate paths in terms of the number of hubs they visit.

\section{Incorporating delivery time restrictions}

In this section, we present a model for the hierarchical hub median problem where the delivery times are bounded above by a constant.

Let $\tau_{i j}$ denote the travel time from node $i \in I$ to node $j \in I$. We assume that $\tau_{i j}=\tau_{j i}$ for all $i$ and $j, \tau_{i i}=0$ for all $i$ and $\tau$ satisfies the triangle inequality. We assume that all the traffic originating at node $i \in I$ is available at node $i$ at time $r_{i}$ and we denote by $\beta$ the time by which all the traffic should reach its destination. There may also be a reduction in travel times between hubs and central hubs and between two central hubs due to the use of specialized vehicles. We denote by $\bar{\alpha}_{H}$ and $\bar{\alpha}_{C}$ these two reduction factors. We assume that both of these parameters take values between 0 and 1 and $\bar{\alpha}_{H} \geqslant \bar{\alpha}_{C}$.

Using the ideas developed in Kara and Tansel (2001) and Wagner (2004), we define the following set of variables. For $l \in C$, let $\hat{D}_{l}$ be the time by which all the traffic from demand nodes and hubs assigned to central hub $l$ arrives at $l$ and let $D_{l}$ be the time at which the traffic with destination at the demand nodes and hubs assigned to central hub $l$ can leave $l$ towards destinations. With these variables, we can model the requirement of transporting all traffic demand to its destinations within the time bound $\beta$ by adding the following constraints to $S A-H-H M$ : 


$$
\begin{aligned}
& \hat{D}_{l} \geqslant \sum_{j \in H}\left(r_{i}+\tau_{i j}+\bar{\alpha}_{H} \tau_{j l}\right) z_{i j l} \quad \forall i \in I, l \in C \\
& D_{l} \geqslant \hat{D}_{k}+\bar{\alpha}_{C} \tau_{k l} z_{k k k} \quad \forall l \in C, \quad k \in C \\
& D_{l}+\sum_{j \in H}\left(\bar{\alpha}_{H} \tau_{l j}+\tau_{j i}\right) z_{i j l} \leqslant \beta \quad \forall i \in I, l \in C \\
& \hat{D}_{l} \geqslant 0 \quad \forall l \in C .
\end{aligned}
$$

If node $i$ is assigned to central hub $l$, then it is assigned to exactly one hub node $j$ which is assigned to central hub $l$. Constraints (22) ensure that the time by which all the traffic from demand nodes and hubs assigned to central hub $l$ arrives at $l$ is no earlier than the time the traffic reaches from $i$ to $l$ (which is equal to the ready time of node $i$ plus the time to travel from $i$ to $l$ through hub $j$ ).

Let $l \in C$ and $k \in C \backslash\{l\}$. First assume that both $l$ and $k$ are central hubs. Then constraint (23) implies that for the traffic with destination at the demand nodes and hubs assigned to central hub $l$ to be ready to leave $l$, the traffic with these destinations should arrive from central hub $k$ to $l$. If $k$ is not a central hub, then constraint (23) implies that $D_{l} \geqslant \hat{D}_{k}$. There exists an optimal solution such that $\hat{D}_{k}=0$ as the right hand sides of constraints (22) imposing lower bounds on $\hat{D}_{k}$ are all zero. Hence this optimal solution satisfies (23). In the remaining case, $k$ is a central hub and $l$ is not. Here constraint (23) still forces $D_{l}$ to be at least as large as $\hat{D}_{k}+\bar{\alpha}_{c} \tau_{k l}$. Suppose that $l$ is assigned to hub $j$ and central hub $m$. Then (23) for $m$ and $k$ implies that $D_{m} \geqslant \hat{D}_{k}+\bar{\alpha}_{c} \tau_{k m}$. Constraint (24) for $m$ and $l$ ensures that $D_{m}+\bar{\alpha}_{H} \tau_{m j}+\tau_{j l} \leqslant \beta$. These together imply that $\hat{D}_{k}+\bar{\alpha}_{C} \tau_{k m}+\bar{\alpha}_{H} \tau_{m j}+\tau_{j l} \leqslant \beta$. Now, since travel times satisfy the triangle inequality, we have $\bar{\alpha}_{C} \tau_{k m}+\bar{\alpha}_{H} \tau_{m j}+\tau_{j l} \geqslant \bar{\alpha}_{C} \tau_{k l}$. Hence we can conclude that there exists an optimal solution satisfying $D_{l} \leqslant \beta$.

Let $l \in C$ and $k=l$. The constraint (23) simplifies to $D_{l} \geqslant \hat{D}_{l}$. This ensures that the vehicles should wait for the traffic originating at the demand nodes and hubs assigned to central hub $l$ to arrive at $l$ to be able to leave $l$ towards these nodes.

As $D_{l}$ is the time at which the traffic with destination at the demand nodes and hubs assigned to central hub $l$ can leave $l, D_{l}+\bar{\alpha}_{H} \tau_{l j}+\tau_{j i}$ is the time that the traffic arrives at its destination at node $i$ if node $i$ is assigned to a hub $j$ that is assigned to the central hub $l$. Constraints (24) ensure that the traffic arrives at node $i$ the latest at time $\beta$.

In telecommunications context, letting $r_{i}=0$ for all $i \in I, \bar{\alpha}_{H}=\bar{\alpha}_{C}=1$ and taking $\tau_{i j}$ equal to the transmission time from node $i$ to node $j$, the above model $S A-T H-H M$ yields a network design where the longest transmission time is bounded above by $\beta$. If different types of equipment are used in hub-to-hub or hub-to-central hub connections, the values of $\bar{\alpha}_{H}$ and $\bar{\alpha}_{C}$ may be adjusted to take into account the differences in transmission speeds.

In this problem, for all origin-destination pairs, we impose the same delivery time restriction. It may be desired to have different restrictions for different origin-destination pairs. Our model cannot handle those restrictions as we do not keep account of the individual origins and destinations in computing the delivery times. A different modeling point of view is required to be able to incorporate such restrictions and this is left as future research.

\section{Computational study}

In our computational study, we use the CAB data and the Turkey data. The CAB data introduced by O'Kelly (1987) is based on the airline passenger traffic between 25 US cities. The data contains the traffic demands and distances. The optimal values are scaled by dividing with the total flow. We take all 25 cities as candidates for hubs and central hubs, i.e., $H=C=I$.

In the Turkey data, the core data is for 81 cities and includes the distances between pairs of cities and the populations. We use the distances as the routing costs. To generate the traffic demand between a pair of cities, we multiply the populations of the two cities, divide by a constant and round down. We denote by $H^{\prime}$ the set of 21 cities where the cargo company has hubs and by $C^{\prime}$ the set of the 10 cities in $H^{\prime}$ with largest populations.

To evaluate the effect of some parameters on the total cost and the locations of central hubs and to see the computation times, we created a dataset taking the most populated 30 cities of the Turkey data as the demand set $I$. We take the intersection of $H^{\prime}$ and $I$ as the set of potential hub locations $H$, and the intersection of $C^{\prime}$ and $I$ as the set of potential central hub locations $C$. In this dataset, the cardinality of set $H$ is 17 and the cardinality of set $C$ is 10 .

\subsection{Effect of the number of central hubs and delivery time restrictions on the total cost}

In our first experiment, we investigate how the total cost is affected by changing the number of central hubs and by imposing delivery time restrictions.

To see the effect of the number of central hubs on the total cost, we use instances from the Turkey data with $n=30$ and $p=7$. In the sequel, $\beta=\infty$ refers to the problem with no time restrictions.

In Figs. 2 and 3, we plot the total costs for different values of $p_{0}$ and $\left(\alpha_{H}, \alpha_{C}\right)$ for $\beta$ equal to $\infty$ and 1920 for the Turkey data. In these instances, $\bar{\alpha}_{C}=\alpha_{C}, \bar{\alpha}_{H}=\alpha_{H}$. The $\left(\alpha_{H}, \alpha_{C}\right)$ values are given on the right of each plot.

We observe that in all cases, for a fixed choice of $\left(\alpha_{H}, \alpha_{C}\right)$, the total cost decreases as we increase $p_{0}$. We see that substantial cost improvements are possible when we move from a star hub network $\left(p_{0}=1\right)$ towards a complete hub network $\left(p_{0}=p\right)$. But clearly, these improvements are in terms of the routing cost and one should consider the trade-off with the cost of operating more central hubs. 


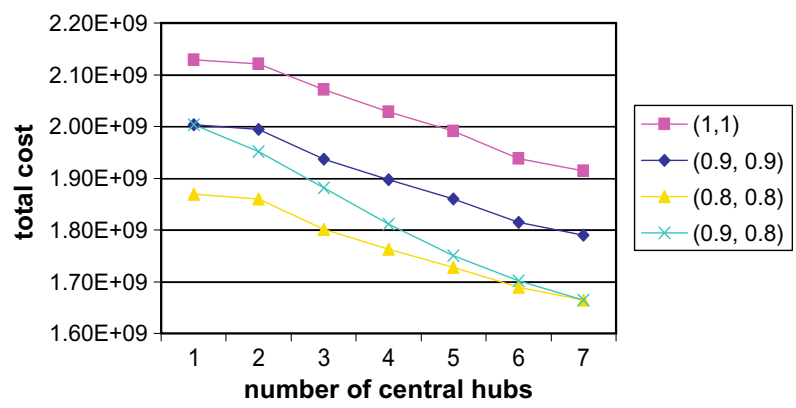

Fig. 2. The total costs for the Turkey data with $n=30, p=7$, and $\beta=\infty$.

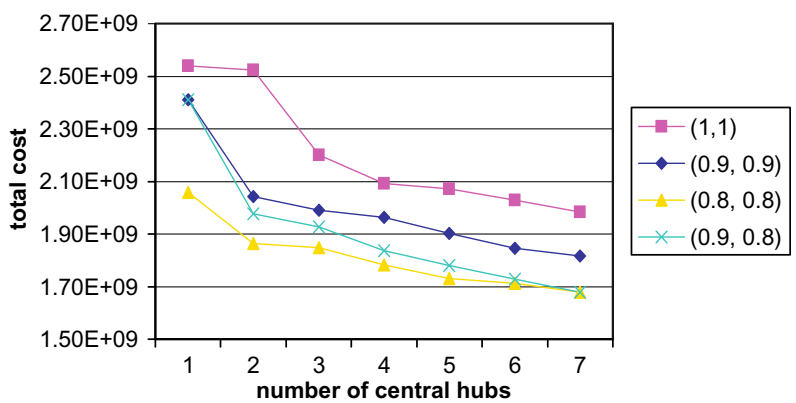

Fig. 3. The total costs for the Turkey data with $n=30, p=7$, and $\beta=1920$.

In Fig. 2, we observe that increasing $p_{0}$ from 1 to 2 does not cause any significant cost reduction when $\alpha_{C}=\alpha_{H}$. Consider an optimal solution with a single central hub. If we pick any hub which is not a central hub in this solution and make it a central hub, then we obtain a feasible solution for the problem with two central hubs. These two solutions have the same cost since $\alpha_{C}=\alpha_{H}$. When we examined the solutions, we saw that when $p_{0}=2$, one of the hubs in the optimal solution for $p_{0}=1$ becomes a central hub and some of the nodes assigned to this node become hubs themselves. But most of the assignments remain the same. As the solutions are very similar and there is no saving in the transportation cost by making a hub a central hub, the improvement in the total cost is small.

In the same plots, we also observe that the discount factors $\left(\alpha_{H}, \alpha_{C}\right)$ have a significant effect on the total cost. Remark that when $p_{0}=1$, as there is a single central hub, there is no routing discounted at the rate $\alpha_{C}$ and when $p_{0}=p$, as all hubs are central hubs, all hub-to-hub routing is discounted at the rate $\alpha_{C}$. Hence, the total cost for $\alpha_{H}=0.9$ and $\alpha_{C}=0.8$ is the same as the one for $\alpha_{H}=\alpha_{C}=0.9$ for $p_{0}=1$ and the same as the one for $\alpha_{H}=\alpha_{C}=0.8$ for $p_{0}=p$. As expected, the effect of increasing the number of central hubs from 1 to $p$ results in a larger decrease when $\alpha_{C}<\alpha_{H}$.

Comparing the plots, we observe that the effect of increasing the number of central hubs on the total cost is higher for the problems where time restrictions are imposed. Actually, the total costs for $p_{0}=p$ with and without time restrictions are very close (the percentage increases are $3.68 \%, 1.45 \%, 0.83 \%$, and $0.83 \%$ for $\left(\alpha_{H}, \alpha_{C}\right)$ equal to $(1,1),(0.9,0.9),(0.8,0.8)$, and $(0.9,0.8)$, respectively). But there is a huge difference when $p_{0}=1$ (the percentage increases are $19.32 \%, 20.4 \%, 10.08 \%$, and $20.4 \%$ for $\left(\alpha_{H}, \alpha_{C}\right)$ equal to $(1,1),(0.9,0.9),(0.8,0.8)$, and $(0.9,0.8)$, respectively). When time restrictions are imposed, some assignments become infeasible and having more possibility in assigning hubs to central hubs has a larger impact on the total cost.

Next, to evaluate the effect of delivery time restrictions on total cost, we performed the following experiment. For the CAB data with $n=25$ and $p=5$ and the Turkey data with $n=30$ and $p=7$, we solved problems where $p_{0} \in\{1, \ldots, p\}$, $\alpha_{H}=0.9, \alpha_{C}=0.8, \bar{\alpha}_{H}=0.9$, and $\bar{\alpha}_{C} \in\{0.9,0.8\}$. For each data set, we computed the smallest $\beta$ values for which the problem is feasible when $\bar{\alpha}_{C}=0.8$ and $\bar{\alpha}_{H}=0.9$. These values differ as we change the number of central hubs. We consider $\beta$ values which are 120 and 240 more than the averages of these smallest values to have a tight and less tight time bound. As a result, we take $\beta \in\{\infty, 2760,2640\}$ for the CAB data and $\beta \in\{\infty, 1920,1800\}$ for the Turkey data.

Suppose that $p_{0}$ and $\bar{\alpha}_{C}$ are fixed. For a given $\beta$, let $v_{\beta}$ be the optimal value of the resulting problem. Then for each $\beta^{\prime}>\beta$, we compute $\%_{\beta^{\prime}}=\frac{v_{\beta}-v_{\beta^{\prime}}}{v_{\beta^{\prime}}} * 100$.

We report the results for the CAB data and the Turkey data in Tables 1 and 2, respectively. We do not report the percentage changes when the problems are infeasible.

The results in these tables show that when $p_{0}$ is small, adding delivery time restrictions to the problem results in considerably higher costs for both data sets. Increasing $p_{0}$ from 1 to 2 results in a large decrease in the percentage increases in costs in almost all cases. 
Table 1

Minimum costs and \% increases in cost for the CAB data with $n=25, p=5$, and $\beta=\infty, 2760,2640$.

\begin{tabular}{|c|c|c|c|c|c|}
\hline \multirow[t]{2}{*}{$\bar{\alpha}_{C}$} & \multirow[t]{2}{*}{$p_{0}$} & \multirow{2}{*}{$\underline{\beta=\infty}$} & \multirow{2}{*}{$\begin{array}{l}\beta=2760 \\
\%_{\infty}\end{array}$} & \multicolumn{2}{|c|}{$\beta=2640$} \\
\hline & & & & $\%_{\infty}$ & $\% 2760$ \\
\hline \multirow[t]{5}{*}{0.8} & 1 & 1200.13 & 15.46 & - & - \\
\hline & 2 & 1146.79 & 3.08 & 7.02 & 3.83 \\
\hline & 3 & 1108.35 & 4.16 & 4.94 & 0.75 \\
\hline & 4 & 1065.06 & 1.96 & 3.39 & 1.40 \\
\hline & 5 & 1034.10 & 1.92 & 2.15 & 0.23 \\
\hline \multirow[t]{5}{*}{0.9} & 1 & 1200.13 & 15.46 & - & - \\
\hline & 2 & 1146.79 & 6.29 & 15.05 & 8.24 \\
\hline & 3 & 1108.35 & 4.94 & 7.32 & 2.27 \\
\hline & 4 & 1065.06 & 3.39 & 6.30 & 2.82 \\
\hline & 5 & 1034.10 & 2.15 & 8.83 & 6.54 \\
\hline
\end{tabular}

Table 2

Minimum costs and \% increases in cost for the Turkey data with $n=30, p=7$, and $\beta=\infty, 1920,1800$

\begin{tabular}{|c|c|c|c|c|c|}
\hline \multirow[t]{2}{*}{$\bar{\alpha}_{C}$} & \multirow[t]{2}{*}{$p_{0}$} & \multirow{2}{*}{$\begin{array}{l}\beta=\infty \\
v_{\infty}\end{array}$} & \multirow{2}{*}{$\begin{array}{l}\beta=1920 \\
\%_{\infty}\end{array}$} & \multicolumn{2}{|c|}{$\beta=1800$} \\
\hline & & & & $\%_{\infty}$ & $\% 1920$ \\
\hline \multirow[t]{7}{*}{0.8} & 1 & 2003005547.4 & 20.40 & 20.63 & 0.19 \\
\hline & 2 & 1951253760.9 & 1.32 & 3.73 & 2.38 \\
\hline & 3 & 1881817611.0 & 2.43 & 2.67 & 0.23 \\
\hline & 4 & 1811525224.4 & 1.36 & 2.43 & 1.06 \\
\hline & 5 & 1750314163.6 & 1.67 & 2.22 & 0.54 \\
\hline & 6 & 1701939783.2 & 1.50 & 1.50 & 0.00 \\
\hline & 7 & 1664450886.0 & 0.83 & 1.57 & 0.73 \\
\hline \multirow[t]{7}{*}{0.9} & 1 & 2003005547.4 & 20.40 & 20.63 & 0.19 \\
\hline & 2 & 1951253760.9 & 2.21 & 5.68 & 3.39 \\
\hline & 3 & 1881817611.0 & 2.67 & 5.18 & 2.44 \\
\hline & 4 & 1811525224.4 & 2.36 & 5.07 & 2.65 \\
\hline & 5 & 1750314163.6 & 2.22 & 5.51 & 3.23 \\
\hline & 6 & 1701939783.2 & 1.50 & 3.71 & 2.17 \\
\hline & 7 & 1664450886.0 & 1.57 & 3.96 & 2.36 \\
\hline
\end{tabular}

For the CAB data, with a single central hub, it is not possible to guarantee a delivery time guarantee of $\beta=2640$ and the total cost increases by $15.46 \%$ when we impose a time limit with $\beta=2760$. When $p_{0}=2$, the problem becomes feasible with $\beta=2640$ but the total cost increases by $15.05 \%$ when $\bar{\alpha}_{C}=0.9$ and by $7.02 \%$ when $\bar{\alpha}_{C}=0.8$. With 4 or 5 central hubs, the increase in the total cost with the inclusion of delivery time limits with $\beta=2760$ remains below $3.5 \%$. For $\bar{\alpha}_{C}=0.8$, we observe that tightening the delivery time restriction from 2760 to 2640 increases the cost by not more than $1.5 \%$ when we have at least three central hubs. Still in most cases, the inclusion of time restrictions results in a significant increase in total costs.

For the Turkey data, when a time limit with $\beta=1920$ is imposed, the increase in the cost is $20.40 \%$ when we have a single central hub but is not more than $3 \%$ when we have more central hubs. However decreasing the bound $\beta$ from 1920 and 1800 results in an increase of only $0.19 \%$ with $p_{0}=1$. This is usually not the case for larger $p_{0}$ values and for $\bar{\alpha}_{C}=0.9$. In this case, even though the increase due to time restrictions with $\beta=1920$ is not very large, we see that the marginal increase remains significant as we tighten the time restrictions.

We can conclude that, for both the $\mathrm{CAB}$ and the Turkey data, imposing delivery time restrictions results in considerable increases in total costs for star hub networks. This result is expected as in a star network, the traffic between two nodes not assigned to the same hub is forced to go through a central hub. In the other extreme case where we use a complete hub network, the traffic between two nodes assigned to two different hubs travels directly between these two hubs. As the triangle inequality is satisfied, traveling between these two hubs by passing through a central hub cannot be shorter than traveling directly. Besides, the travel times between two hubs and a central hub are reduced by the factor $\bar{\alpha}_{H}$ in the star hub network whereas the travel times between two central hubs are reduced by the factor $\bar{\alpha}_{C}$ in the complete hub network. Hence, for the same assignment of demand nodes to hubs, the distances between origin-destination pairs are likely to be longer in a star hub network compared to a complete hub network.

\subsection{Effect of the number of central hubs, the discount factors, and delivery time restrictions on the locations of central hubs}

In this experiment, we would like to observe the effect of the number of central hubs, the discount factors, and the delivery time restrictions on the locations of central hubs. For this, we use the CAB data with $n=25, p=5, p_{0}=2,3,4$, and $\beta=\infty, 2760,2640$ and the Turkey data with $n=30, p=7, p_{0}=3,4,5, \beta=\infty, 1920,1800$, and different discount factors. In Tables 3 and 4, we report the locations of hubs and central hubs in the optimal solutions for these instances. 
Table 3

The locations of hubs and central hubs in the optimal solutions for the CAB data with $n=25$ and $p=5$.

\begin{tabular}{|c|c|c|c|c|}
\hline$\left(\alpha_{h}, \alpha_{C}, \bar{\alpha}_{H}, \bar{\alpha}_{C}\right)$ & $\beta$ & $p_{0}$ & Central hubs & Hubs \\
\hline \multirow[t]{5}{*}{$(1,1,1,1)$} & $\infty$ & 2 & 4,20 & $4,8,17,20,21$ \\
\hline & & 3 & $4,20,21$ & $4,8,17,20,21$ \\
\hline & & 4 & $4,7,8,20$ & $4,7,8,17,20$ \\
\hline & 2760 & 3 & $8,11,23$ & $6,8,11,13,23$ \\
\hline & & 4 & $4,8,13,23$ & $4,6,8,13,23$ \\
\hline \multirow[t]{9}{*}{$(0.9,0.9,0.9,0.9)$} & $\infty$ & 2 & 4,20 & $4,8,17,20,21$ \\
\hline & & 3 & $4,20,21$ & $4,12,17,20,21$ \\
\hline & & 4 & $1,4,11,18$ & $1,4,11,12,18$ \\
\hline & 2760 & 2 & 8,21 & $8,12,20,21,24$ \\
\hline & & 3 & $4,8,13$ & $4,8,13,18,24$ \\
\hline & & 4 & $4,8,13,20$ & $4,8,13,17,20$ \\
\hline & 2640 & 2 & 8,11 & $6,8,11,23,24$ \\
\hline & & 3 & $4,8,13$ & $4,8,13,17,23$ \\
\hline & & 4 & $4,8,13,20$ & $4,8,13,20,23$ \\
\hline \multirow[t]{9}{*}{$(0.8,0.8,0.8,0.8)$} & $\infty$ & 2 & 4,20 & $4,12,17,20,24$ \\
\hline & & 3 & $4,20,21$ & $4,12,17,20,21$ \\
\hline & & 4 & $1,4,11,18$ & $1,4,11,12,18$ \\
\hline & 2760 & 2 & 5,8 & $5,8,13,18,24$ \\
\hline & & 3 & $4,8,13$ & $4,8,12,13,17$ \\
\hline & & 4 & $1,4,8,18$ & $1,4,8,12,18$ \\
\hline & 2640 & 2 & 8,21 & $8,12,20,21,24$ \\
\hline & & 3 & $4,8,13$ & $4,8,12,13,18$ \\
\hline & & 4 & $4,8,13,18$ & $4,8,12,13,18$ \\
\hline \multirow[t]{9}{*}{$(0.9,0.8,0.9,0.8)$} & $\infty$ & 2 & 4,20 & $4,8,17,20,21$ \\
\hline & & 3 & $4,12,20$ & $4,12,17,20,21$ \\
\hline & & 4 & $4,7,12,20$ & $4,7,12,17,20$ \\
\hline & 2760 & 2 & 5,8 & $5,8,13,14,18$ \\
\hline & & 3 & $5,7,8$ & $5,7,8,14,18$ \\
\hline & & 4 & $1,2,4,8$ & $1,2,4,8,17$ \\
\hline & 2640 & 2 & 8,21 & $8,20,21,23,24$ \\
\hline & & 3 & $8,20,21$ & $8,17,20,21,24$ \\
\hline & & 4 & $1,4,8,18$ & $1,4,8,18,23$ \\
\hline \multirow[t]{9}{*}{$(0.9,0.8,0.9,0.9)$} & $\infty$ & 2 & 4,20 & $4,8,17,20,21$ \\
\hline & & 3 & $4,12,20$ & $4,12,17,20,21$ \\
\hline & & 4 & $4,7,12,20$ & $4,7,12,17,20$ \\
\hline & 2760 & 2 & 8,21 & $8,12,20,21,24$ \\
\hline & & 3 & $8,20,21$ & $8,17,20,21,24$ \\
\hline & & 4 & $1,4,8,18$ & $1,4,8,18,23$ \\
\hline & 2640 & 2 & 8,11 & $6,8,11,23,24$ \\
\hline & & 3 & $4,8,13$ & $4,8,13,17,23$ \\
\hline & & 4 & $4,8,13,17$ & $4,8,13,17,23$ \\
\hline
\end{tabular}

We first study the results for the CAB data. When $\beta=\infty$, city 4 appears as a central hub in all instances. When $p_{0}=2$, cities 4 and 20 are the central hubs for all different values of discount factors considered. They also appear as central hubs when an additional central hub is opened. In this case, city 21 becomes the third central hub in the instances with $\alpha_{C}=\alpha_{H}$. In the instance with $\alpha_{C}<\alpha_{H}$, the third hub moves west and is opened at city 12 . As $p_{0}$ increases to 4 , we observe that the locations of central hubs become more dispersed.

To see the effect of decreasing the value of the discount factor for the transportation cost among central hubs, we compare the results for the instances with $\alpha_{C}=\alpha_{H}=0.9$ and $\alpha_{C}=0.8, \alpha_{H}=0.9$. When $\beta=\infty$, for $p_{0}=2$, the central hubs remain the same for $\alpha_{C}=0.9$ and $\alpha_{C}=0.8$. As we have mentioned above, for $p_{0}=3$, the central hub at city 21 moves to city 12 when $\alpha_{C}$ decreases from 0.9 to 0.8 . For $p_{0}=4$, the central hubs located at cities 1,11 , and 18 move to cities 7,12 , and 20 . Hence, we observe that central hubs move to cities which have higher traffic demands as the discount factor decreases in these instances. However, comparing the locations of central hubs for $\alpha_{H}=\alpha_{C}=1$ and $\alpha_{H}=\alpha_{C}=0.8$ with $\beta=\infty$ shows that such a conclusion cannot be generalized. When a delivery time restriction is imposed with $\beta=2760$, for $p_{0}=2$, the central hub at city 8 remains but the one located at city 21 moves to city 5 which is further away than city 8 . For $p_{0}=3$, the central hubs at cities 4 and 13 move to cities 5 and 7 , and for $p_{0}=4$, the central hubs at cities 13 and 20 move to cities 1 and 2 . These results suggest that the central hubs move away from each other and the center of the country when $\alpha_{C}<\alpha_{H}$.

We observe that imposing delivery time restrictions can have a large impact on the locations of central hubs in the optimal solutions. For instance, when all discount factors are 1 and $p_{0}=3$, cities 4,20 , and 21 are central hubs for $\beta=\infty$ and cities 8,11 , and 23 are central hubs for $\beta=2760$. Not only all central hubs move but also they move for long distances.

When there are no discounts and no delivery time restrictions, the hubs are located at cities 4, 8, 17, 20, and either 7 or 21 . For $p_{0}=2,3,4$, cities 4 and 20 are chosen as central hubs. The most western city used as a central hub here is city 8 and is 
Table 4

The locations of hubs and central hubs in the optimal solutions for instances with $n=30$ and $p=7$.

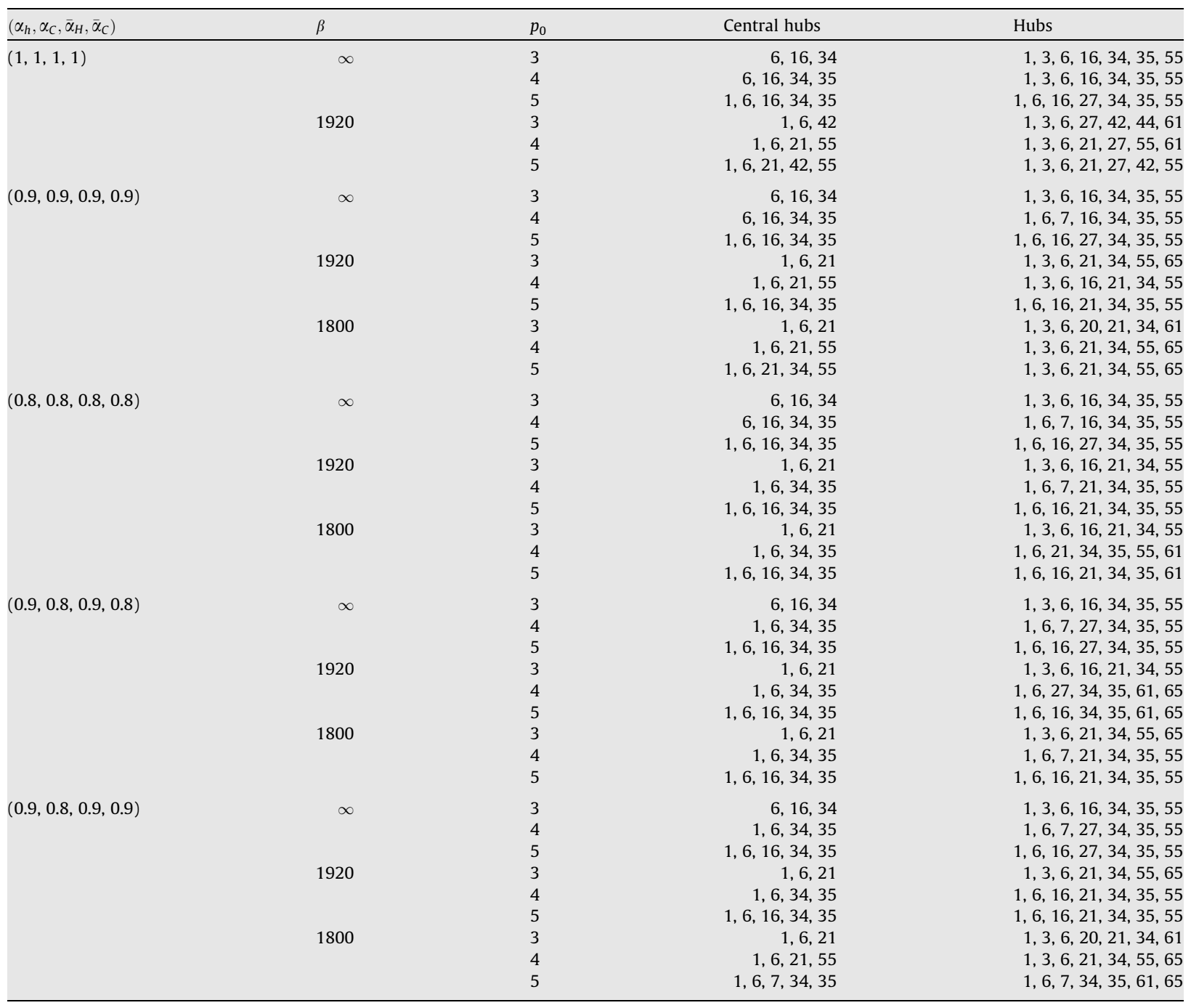

closer to the center. When a delivery time restriction is imposed with $\beta=2760$, cities 8 and 23 become central hubs for $p_{0}=3$ and 4 . Here we observe that central hubs move west with the addition of these restrictions. Note here that city 23, which is located at the northwest corner of the country and is quite far away from all other cities in this data set, is picked as a central hub only when there are no discounts and delivery time restrictions are imposed. It is sometimes used as a hub but again only when there are time restrictions.

The union of the set of central hubs over instances with $\beta=\infty$ is the set of cities $1,4,7,8,11,12,18,20$, and 21 . These cities are mostly situated in the central and eastern parts of the country except 12 . City 4 is used in all 12 instances and city 20 is used in 10 instances. Others are not commonly used. Cities $1,7,8,11$, and 18 are central hubs only when $p_{0}=4$, and city 21 is a central hub only when $p_{0}=3$. Finally, a central hub is located at city 12 when $\alpha_{C}<\alpha_{H}$ and $p_{0}=3$, 4. Cities 17 and 24 appear as hubs but never as central hubs.

When we impose delivery time restrictions, cities $2,5,13,17$, and 23 also appear as central hubs. City 12 is not used as a central hub any more. City 8 is used in all 26 instances. Cities 4 and 13 are also commonly used when $p_{0}$ is 3 and 4. Cities 2, 7, and 17 appear as central hubs only once. Hubs are located at cities 6,12,14, and 24 in some instances but central hubs are not located at these cities.

In Table 4, we report the locations of hubs and central hubs in the optimal solutions for the Turkey data. We use the twodigit license plate codes to refer to the cities. To be able to visualize the changes in the locations of hubs and central hubs, in Fig. 4, we give a map of Turkey where the dots represent the 30 nodes that are the demand centers for the instances used in this experiment. The squares are used to represent the demand centers that are candidates to become central hubs and the circles are used to represent the demand centers that are candidates to become hubs but not central hubs. We display the license plate codes for candidate nodes to becomes hubs or central hubs. 


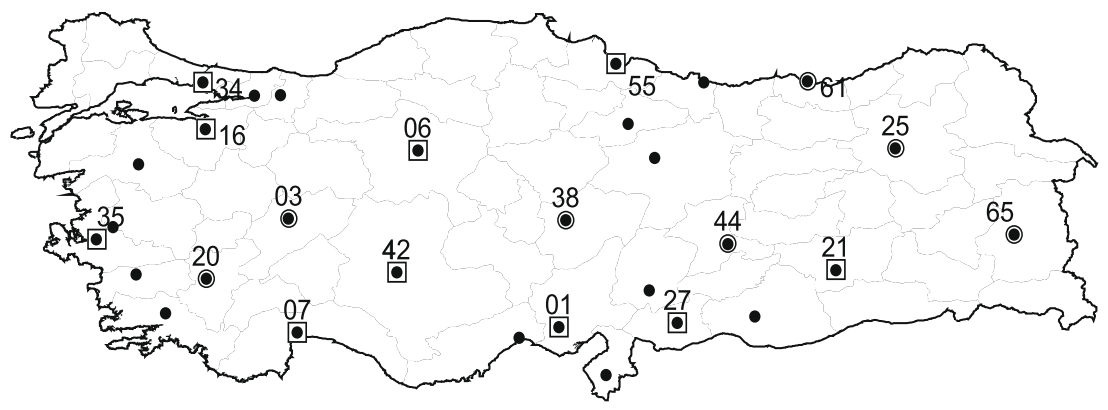

Fig. 4. The map for the 30 node instance.

First we notice that for the same values of $\beta$ and discount factors, in most cases, the central hubs in an optimal solution for a fixed $p_{0}$ also appear as central hubs for $p_{0}+1$. For instance for $\beta=\infty$, nodes 6,16 , and 34 are central hubs for $p_{0}=3$ and all values of $\left(\alpha_{H}, \alpha_{C}\right)$. When $p_{0}=4$, node 35 also becomes a central hub. Only when $\alpha_{H}=0.9$ and $\alpha_{C}=0.8$, node 16 is replaced with node 1 . Later when we increase $p_{0}$ to 5 , both cities 1 and 16 are central hubs for all values of $\left(\alpha_{H}, \alpha_{C}\right)$.

Node 6 is chosen as a central hub and node 1 is chosen as a hub or central hub in optimal solutions for all instances. Same nodes appear as hubs and central hubs in optimal solutions for most instances. When $p_{0}$ is large, the locations of hubs and central hubs remain mostly the same when delivery time restrictions are imposed.

We observe that when $\beta=\infty$, the locations of hubs and central hubs remain the same for different values of $\left(\alpha_{h}, \alpha_{C}\right)$ except for the instance with $p_{0}=4, \alpha_{H}=0.9$ and $\alpha_{C}=0.8$. We also observe that nodes 6 (Ankara) and 34 (Istanbul) appear as central hubs in all these instances. These are the two most populated cities of Turkey. As we increase $p_{0}$, the third most populated city Izmir (node 35) also becomes a central hub. Actually, the central hubs are chosen from the first six most populated cities. Only Konya (node 42) in the first six most populated cities is not a central hub for $p_{0}=5$, probably because it is close to Ankara.

For $\beta=\infty$, the locations of the central hubs are not affected by the changes in the discount parameters except for the case with $p_{0}=4$. Indeed, the optimal locations for problems without time restrictions seem less sensitive to the changes in discount parameters. Other than this instance, in general, we observed that central hubs move to more populated cities as the discount factors decreased. For instance when $p_{0}=3$ and $\beta=2040$, the central hub at city 55 (the 14 th most populated city) is replaced with a central hub at city 21 (the 10th most populated city) as $\alpha_{C}$ and $\alpha_{H}$ decrease from 1 to 0.9 .

We considered also the combination $(0.9,0.8,0.9,0.9)$, to see the effect of having different values for $\alpha_{C}$ and $\bar{\alpha}_{C}$. When we compared the optimal solutions for this combination to those for $(0.9,0.9,0.9,0.9)$, we observed that either the central hubs remained the same or moved to more populated cities.

Finally, when $\bar{\alpha}_{H}$ and $\bar{\alpha}_{C}$ are large and $\beta$ small, we observe that central hubs move away from the center of population. Nodes 21 (Diyarbakir) and 55 (Samsun) are commonly used as central hubs for the instances with tight delivery time restrictions. Notice that both cities were not central hubs in any of the instances with no delivery time restrictions. Diyarbakir is located in the south-eastern part and Samsun is located in the northern part of the country and are highly populated cities in their regions.

Hence, we again observe that central hubs move away from the center to be able to satisfy delivery time restrictions for extremal origin-destination pairs.

When we compare the results for the CAB data and the Turkey data, we observe some similarities and difference. The fact that some nodes appear as central hubs in most solutions is a common property. Such nodes are mostly centrally located or have large demand or are at the center of nodes with large demand. As the discount factors decrease, central hubs move to more populated cities and when tight time restrictions are imposed, central hubs move away from the center.

For the Turkey instances with large number of central hubs, the locations of hubs and central hubs do not change much when delivery time restrictions are imposed. We have not observed a similar result for the CAB data. There may be several reasons for this. First of all, with the Turkey data, we take $p=7$ whereas for the CAB data, we took $p=5$. Moreover, in the Turkey data, we restrict the set of hubs and central hubs to subsets of the node set. We also observe that cities used in the dataset are mostly concentrated on the eastern part of the US in the CAB data whereas concentration at a specific part of the country is less apparent in the Turkey data. Finally, the locations of the central hubs and hubs are more dispersed in the Turkey instances already without the time restrictions.

\subsection{Computation times}

In this section, we investigate how the computation times are affected by the parameters of the problem. All instances are solved using GAMS 22.5 and CPLEX 11.0.0 on an AMD Opteron 252 processor (2.6 GHz) with 2 GB of RAM operating under the system CentOS (Linux version 2.6.9-42.0.3.ELsmp). For the instances with $\beta=\infty$, we solve the model (10)-(21), and for the instances with delivery time restrictions, we solve the model (10)-(25). 
In Table 5, we report the results for the CAB data with $n=25$ and $p=5$. We do not report the computation times for the infeasible instances. We observe that the instances with $p_{0}=p$ are the easiest instances. The most difficult instances are those with $p_{0}=2,3,4$. The longest computation time is close to $20 \mathrm{~h}$. Except for the instances with $p_{0}=1$, problems with delivery time restrictions take less time to solve to optimality. The instance with $\left(\alpha_{h}, \alpha_{C}, \bar{\alpha}_{H}, \bar{\alpha}_{C}\right)=(0.9,0.8,0.9,0.8)$ and $p_{0}=2$ is solved in $61561.77 \mathrm{~s}$ without time restrictions and in 1916.61 seconds when time restrictions with $\beta=2760$ are imposed, around 32 times faster.

In Table 6, we report the results for the Turkey data with $n=30$ and $p=7$. We observe that the instances with $p_{0}$ equal to 1 and $p$ are the easiest instances to solve and $p_{0}$ equal to 2, 3, 4, and 5 are the hardest instances to solve. As in the case of the $\mathrm{CAB}$ data, adding delivery time restrictions result in shorter computation times in most cases.

\subsection{The problem with two central hubs located in Ankara and Istanbul}

As a benchmark with the company's current network, we solve the problem with 81 cities where we set $p_{0}=2$ and fix the locations of the central hubs to Ankara and Istanbul, $\alpha_{C}=\bar{\alpha}_{C}=0.8$ and $\alpha_{H}=\bar{\alpha}_{H}=0.9$. The reason for taking $\bar{\alpha}_{H}=0.9$ is that larger trucks operate between hubs and central hubs and travel around 1.1 faster than smaller trucks that operate between demand centers and hubs (see also Tan and Kara (2007) and Yaman et al. (2007)). We took $\bar{\alpha}_{C}=0.8$ to reflect both the saving in time due to the use of larger trucks and due to the fact that a highway connection exists between Ankara and Istanbul. We vary $p$ from 7 to 12 .

In Fig. 5, we give the Turkey map with the 81 cities. We use circles to represent the demand centers that are candidates to become hubs. We also display the license plate codes for these nodes. The two black squares correspond to Ankara and Istanbul.

We computed the minimum value of $\beta$ for which the problem has a feasible solution. This value turned out to be 2589.6 min for all the $p$ values considered in this experiment. We report the results for $\beta$ equal to this minimum value and infinity and every other value of $\beta$ that is greater than 2589.6 , divisible by 60 , and for which there is no smaller $\beta$ with the same optimal value. These results are given in Table 7.

In Figs. 6 and 7, we give the maps with the locations of hubs for the instances with $p=7$ and $\beta$ equal to infinity and 2640 . The full circles are the hubs in the optimal solution.

When $\beta=\infty$ and $p=7$, we observe that three hubs are located at cities that lie between Istanbul and Ankara. These cities cover the most populated and industrialized region of the country. Then one hub is located at the northern part of the country at city 55 which has the highest population among the northern cities. The remaining hub is located at city 38 which covers the southeastern part of the country. In general without delivery time restrictions, hubs are located in larger cities. The only exception is the city 81 which ranks the 60th in terms of population. This city has a hub in most of the solutions

Table 5

Computation times in seconds for the CAB data with $n=25$ and $p=5$.

\begin{tabular}{|c|c|c|c|c|}
\hline$\left(\alpha_{h}, \alpha_{C}, \bar{\alpha}_{H}, \bar{\alpha}_{C}\right)$ & $p_{0}$ & $\infty$ & 2760 & 2640 \\
\hline \multirow[t]{5}{*}{$(1,1,1,1)$} & 1 & 477.38 & - & - \\
\hline & 2 & 19355.87 & - & - \\
\hline & 3 & 12755.77 & 1006.25 & - \\
\hline & 4 & 26657.54 & 1000.79 & - \\
\hline & 5 & 288.00 & 16.06 & - \\
\hline \multirow[t]{5}{*}{$(0.9,0.9,0.9,0.9)$} & 1 & 685.46 & 1865.86 & - \\
\hline & 2 & 35826.54 & 2703.06 & 1769.03 \\
\hline & 3 & 20098.73 & 9525.84 & 1878.56 \\
\hline & 4 & 70216.72 & 5295.32 & 1130.82 \\
\hline & 5 & 253.02 & 73.02 & 53.45 \\
\hline \multirow[t]{5}{*}{$(0.8,0.8,0.8,0.8)$} & 1 & 1642.01 & 2733.11 & 2333.87 \\
\hline & 2 & 12126.83 & 6868.51 & 6128.63 \\
\hline & 3 & 17357.99 & 6632.61 & 5014.60 \\
\hline & 4 & 34253.30 & 3956.04 & 5182.10 \\
\hline & 5 & 150.06 & 154.80 & 159.87 \\
\hline \multirow[t]{5}{*}{$(0.9,0.8,0.9,0.8)$} & 1 & 635.74 & 2388.69 & - \\
\hline & 2 & 61561.77 & 1916.61 & 2128.85 \\
\hline & 3 & 51699.33 & 7714.07 & 5591.63 \\
\hline & 4 & 19831.40 & 4690.15 & 5439.34 \\
\hline & 5 & 141.30 & 155.63 & 164.27 \\
\hline \multirow[t]{5}{*}{$(0.9,0.8,0.9,0.9)$} & 1 & 635.74 & 2014.60 & - \\
\hline & 2 & 61561.77 & 2860.42 & 2002.66 \\
\hline & 3 & 51699.33 & 8329.49 & 1631.86 \\
\hline & 4 & 19831.40 & 4903.33 & 2031.89 \\
\hline & 5 & 141.30 & 119.80 & 63.37 \\
\hline
\end{tabular}


Table 6

Computation times in seconds for the Turkey data with $n=30$ and $p=7$.

\begin{tabular}{|c|c|c|c|c|}
\hline$\left(\alpha_{h}, \alpha_{C}, \bar{\alpha}_{H}, \bar{\alpha}_{C}\right)$ & $p_{0}$ & $\infty$ & 1920 & 1800 \\
\hline \multirow[t]{7}{*}{$(1,1,1,1)$} & 1 & 158.97 & 142.32 & - \\
\hline & 2 & 8303.42 & 117.77 & - \\
\hline & 3 & 8590.76 & 137.89 & - \\
\hline & 4 & 2416.36 & 95.72 & - \\
\hline & 5 & 2204.42 & 116.67 & - \\
\hline & 6 & 511.10 & 133.04 & - \\
\hline & 7 & 11.35 & 8.69 & - \\
\hline \multirow[t]{7}{*}{$(0.9,0.9,0.9,0.9)$} & 1 & 53.42 & 122.85 & 98.73 \\
\hline & 2 & 2813.86 & 119.20 & 92.87 \\
\hline & 3 & 2114.43 & 191.66 & 127.49 \\
\hline & 4 & 1919.72 & 304.30 & 110.59 \\
\hline & 5 & 1052.36 & 225.54 & 134.33 \\
\hline & 6 & 432.10 & 176.47 & 117.06 \\
\hline & 7 & 3.55 & 7.50 & 6.98 \\
\hline \multirow[t]{7}{*}{$(0.8,0.8,0.8,0.8)$} & 1 & 43.94 & 107.63 & 80.62 \\
\hline & 2 & 2361.28 & 144.61 & 109.18 \\
\hline & 3 & 1683.54 & 646.48 & 291.52 \\
\hline & 4 & 1100.92 & 583.55 & 187.32 \\
\hline & 5 & 665.27 & 117.67 & 161.69 \\
\hline & 6 & 420.38 & 237.51 & 141.25 \\
\hline & 7 & 2.80 & 5.95 & 6.46 \\
\hline \multirow[t]{7}{*}{$(0.9,0.8,0.9,0.8)$} & 1 & 38.12 & 123.55 & 134.95 \\
\hline & 2 & 1553.45 & 164.73 & 151.43 \\
\hline & 3 & 4061.13 & 338.59 & 181.98 \\
\hline & 4 & 1101.86 & 241.36 & 156.40 \\
\hline & 5 & 535.32 & 245.79 & 154.74 \\
\hline & 6 & 131.97 & 214.41 & 85.26 \\
\hline & 7 & 2.86 & 5.78 & 6.30 \\
\hline \multirow[t]{7}{*}{$(0.9,0.8,0.9,0.9)$} & 1 & 38.12 & 127.16 & 102.37 \\
\hline & 2 & 1553.45 & 82.32 & 140.09 \\
\hline & 3 & 4061.13 & 216.24 & 140.31 \\
\hline & 4 & 1101.86 & 218.88 & 125.88 \\
\hline & 5 & 535.32 & 146.56 & 134.32 \\
\hline & 6 & 131.97 & 101.82 & 132.83 \\
\hline & 7 & 2.86 & 6.51 & 4.61 \\
\hline
\end{tabular}

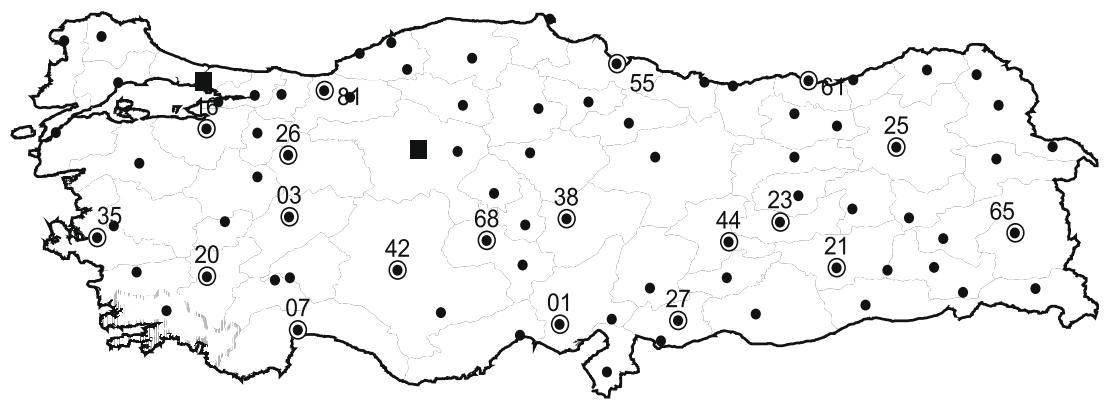

Fig. 5. The Turkey map.

since it is very close to city 41 which is the 15 th most populated city but is not a candidate to become a hub since the cargo company does not have a hub here.

We observe that when a delivery time restriction with $\beta=2640$ is introduced, the three hubs two of which lie between Ankara and Istanbul and the remaining one close to Ankara are replaced with hubs at cities more in the southern and eastern parts of the country. As the longest distances exist between the east and the west of Turkey and there is already a central hub located in Istanbul, such a move of hubs towards the east is not surprising. The two hubs which remain at cities 3 and 55 are located in the western and northern parts of the country. The three new hubs are now at city 1 located in the south, 25 in the northeast, and 44 in the southeast. These cities are also highly populated cities in their regions.

In Fig. 8, we depict the hub network for $p=10$ and $\beta=\infty$. The locations of hubs remain the same when we add delivery time restrictions with $\beta$ equal to 2700 and 2640 . When we set a time restriction of $\beta$ equal to 2589.6, the hub at city 42 is replaced with a hub at city 21 . 
Table 7

The results for 81 cities.

\begin{tabular}{|c|c|c|c|c|}
\hline$p$ & $\beta$ & Cost & Hubs & cpu \\
\hline \multirow[t]{4}{*}{7} & $\infty$ & 4056967296.4 & $3,6,26,34,38,55,81$ & 1190.28 \\
\hline & 2700 & 4056967296.4 & $3,6,26,34,38,55,81$ & 624.31 \\
\hline & 2640 & 4057885668.0 & $1,3,6,25,34,44,55$ & 422.48 \\
\hline & 2589.6 & 4083832304.2 & $1,3,6,21,25,34,55$ & 322.89 \\
\hline \multirow[t]{4}{*}{8} & $\infty$ & 4046334302.0 & $1,3,6,16,25,34,44,55$ & 2757.05 \\
\hline & 2700 & 4046334302.0 & $1,3,6,16,25,34,44,55$ & 1604.65 \\
\hline & 2640 & 4046448524.4 & $1,3,6,16,25,34,44,55$ & 5386.48 \\
\hline & 2589.6 & 4067051053.2 & $1,3,6,21,25,34,44,55$ & 152.90 \\
\hline \multirow[t]{4}{*}{9} & $\infty$ & 4037395948.6 & $1,3,6,25,26,34,44,55,81$ & 10132.97 \\
\hline & 2700 & 4037395948.6 & $1,3,6,25,26,34,44,55,81$ & 859.22 \\
\hline & 2640 & 4037510171.0 & $1,3,6,25,26,34,44,55,81$ & 431.94 \\
\hline & 2589.6 & 4054521997.6 & $1,3,6,16,21,25,34,44,55$ & 230.99 \\
\hline \multirow[t]{4}{*}{10} & $\infty$ & 4028897663.4 & $1,3,6,25,26,34,42,44,55,81$ & 6118.10 \\
\hline & 2700 & 4028897663.4 & $1,3,6,25,26,34,42,44,55,81$ & 866.00 \\
\hline & 2640 & 4029011885.8 & $1,3,6,25,26,34,42,44,55,81$ & 414.24 \\
\hline & 2589.6 & 4045583644.2 & $1,3,6,21,25,26,34,44,55,81$ & 557.33 \\
\hline \multirow[t]{4}{*}{11} & $\infty$ & 4024370239.4 & $1,3,6,25,26,34,38,42,44,55,81$ & 6136.92 \\
\hline & 2700 & 4024370239.4 & $1,3,6,25,26,34,38,42,44,55,81$ & 513.21 \\
\hline & 2640 & 4024484461.8 & $1,3,6,25,26,34,38,42,44,55,81$ & 655.92 \\
\hline & 2589.6 & 4037085359.0 & $1,3,6,21,25,26,34,42,44,55,81$ & 811.84 \\
\hline \multirow[t]{3}{*}{12} & $\infty$ & 4021762961.6 & $1,3,6,16,25,26,34,38,42,44,55,81$ & 16786.83 \\
\hline & 2640 & 4021762961.6 & $1,3,6,16,25,26,34,38,42,44,55,81$ & 851.13 \\
\hline & 2589.6 & 4032557935.0 & $1,3,6,21,25,26,34,38,42,44,55,81$ & 423.04 \\
\hline
\end{tabular}

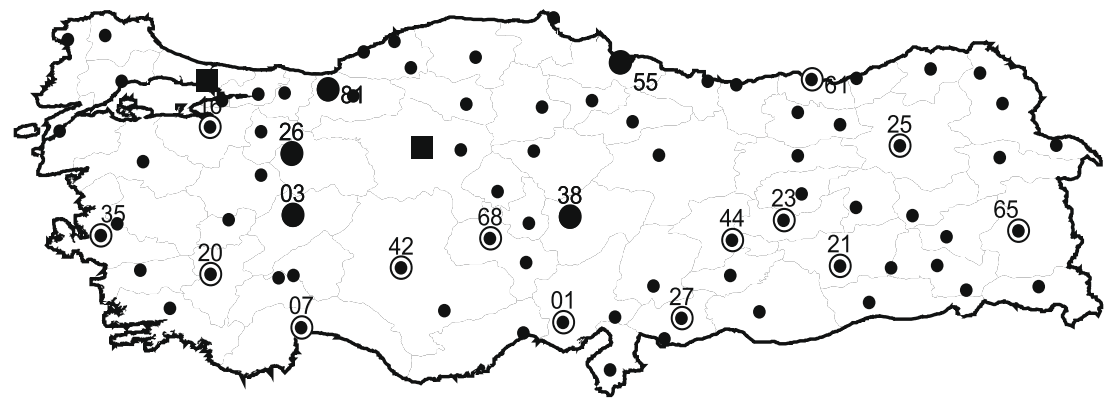

Fig. 6. The hub network for $n=81, \alpha_{C}=0.8, \alpha_{H}=0.9, p=7$, and $\beta=\infty$.

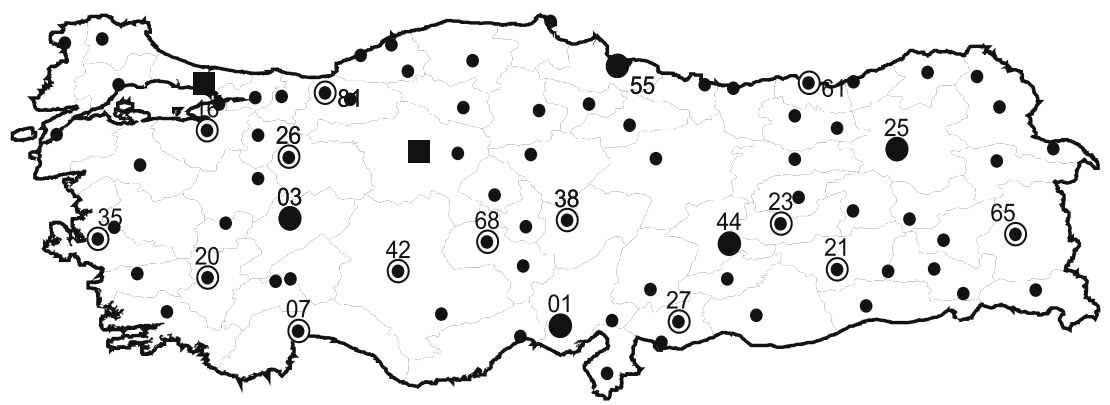

Fig. 7. The hub network for $n=81, \alpha_{C}=0.8, \alpha_{H}=0.9, p=7$, and $\beta=2640$.

For $p=11$, we observe that the hub at city 38 is replaced with a hub at city 21 and for $p=12$, the hub at city 16 is replaced with a hub at city 21 when a delivery time restriction with $\beta=2589.6$ is imposed. Hence some hubs located close to central hubs are moved to further cities when a tight time restriction is forced. We also remark that time restrictions result in more changes in the locations of hubs for $p=7$ compared to larger values of $p$.

We analyzed the assignment of hubs to central hubs in the optimal solutions to evaluate the number of loading/unloading operations to be performed on a path connecting an origin-destination pair. We observed that all hubs other than Istanbul are assigned to the central hub at Ankara. Hence a path between an origin-destination pair visits at most three hubs. 


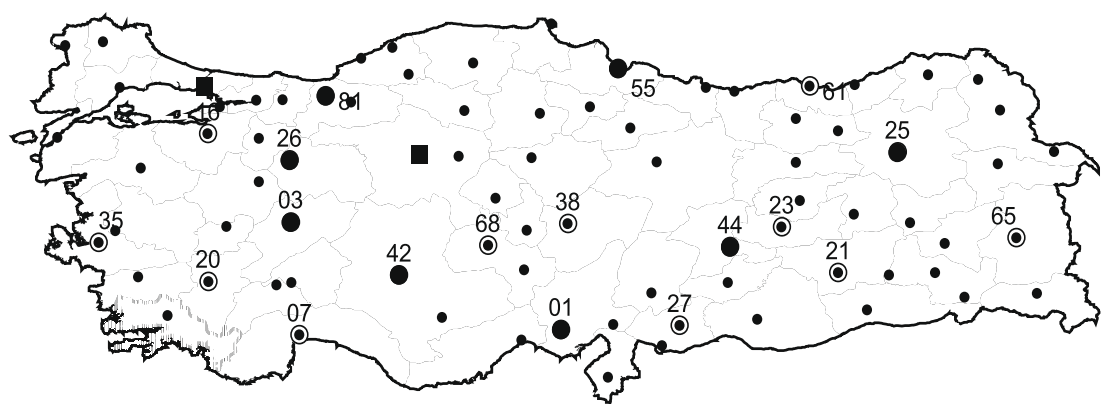

Fig. 8. The hub network for $n=81, \alpha_{C}=0.8, \alpha_{H}=0.9, p=10$, and $\beta=\infty$.

As a final remark, we note that except for $p=8$, the solver spent much more time to solve the problems with $\beta=\infty$ compared to the problems with finite $\beta$.

\section{Conclusion}

In this paper, we introduced a hierarchical hub location problem and presented a mixed integer programming model to solve it. The $p$-hub median problem and the star $p$-hub median problem turned out to be two special cases of our problem. More precisely, the $p$-hub median problem is a special case where the number of central hubs is equal to the number of hubs and the star $p$-hub median problem is a special case where the number of central hubs to be opened is equal to 1 and the candidate set is a singleton. By solving our problem for the same instance with the number of central hubs varying from 1 to $p$, we could investigate the effect of using central hubs on the total cost.

To be able to take into consideration the service quality in the design process, we incorporated delivery time restrictions to our hierarchical hub location problem. We solved our instances with different levels of time restrictions and investigated the effect of these restrictions in the total routing costs and locations of central hubs.

In general, we observed that the effect of delivery time restrictions were stronger when the number of central hubs and hubs were smaller. For a given number of hubs, we observed a smaller increase in total cost due to delivery time restrictions as we moved from the star hub network towards the complete hub network. We also observed that some of the hubs moved away from the central hubs when time restrictions are imposed.

From the computational point of view, the problems with delivery time restrictions turned out to be easier to solve compared to the problems with no time restrictions.

\section{Acknowledgement}

We are grateful to an anonymous referee for his/her comments and suggestions which improved the content and the presentation of the results. This research is partially supported by TUBITAK Grant No. 107M460.

\section{References}

Abdinnour-Helm, S., 2001. Using simulated annealing to solve the $p$-hub median problem. International Journal of Physical Distribution and Logistics Management $31(3), 203-220$.

Alumur, S., Kara, B.Y., 2008. Network hub location problems: the state of the art. European Journal of Operational Research 190 (1), 1-21.

Campbell, J.F., 1994. Integer programming formulations of discrete hub location problems. European Journal of Operational Research 72 (2), 387-405

Campbell, J.F., 1996. Hub location and the $p$-hub median problem. Operations Research 44 (6), 923-935.

Campbell, J.F., Ernst, A.T., Krishnamoorthy, M., 2002. Hub location problems. In: Drezner, Z., Hamacher, H. (Eds.), Facility Location: Applications and Theory. Springer-Verlag, Berlin.

Ebery, J., 2001. Solving large single allocation $p$-hub problems with two or three hubs. European Journal of Operational Research 128 (2), 447-458.

Elmastas, S., 2006. Hub location problem for air-ground transportation systems with time restrictions. M.S. Thesis, Bilkent University, Department of Industrial Engineering.

Ernst, A.T., Krishnamoorthy, M., 1996. Efficient algorithms for the uncapacitated single allocation p-hub median problem. Location Science 4 (3), $139-154$.

Ernst, A.T., Krishnamoorthy, M., 1998. An exact solution approach based on shortest paths for $p$-hub median problems. INFORMS Journal on Computing 10 (2), 149-162.

Ernst, A.T., Jiang, H., Krishnamoorthy, M., 2005. Reformulations and computational results for uncapacitated single and multiple allocation hub covering problems. Unpublished Report, CSIRO Mathematical and Information Sciences, Australia.

Kara, B.Y., Tansel, B.C., 2001. The latest arrival hub location problem. Management Science 47 (10), 1408-1420.

Kara, B.Y., Tansel, B.C., 2003. The single assignment hub covering problem: models and linearizations. Journal of the Operational Research Society 54 (1), 5964.

Klincewicz, J.G., 1991. Heuristics for the p-hub location problem. European Journal of Operational Research 53 (1), 25-37.

Klincewicz, J.G., 1992. Avoiding local optima in the $p$-hub location problem using tabu search and GRASP. Annals of Operations Research 40 (1), $283-302$.

Labbé, M., Yaman, H., 2008. Solving the hub location problem in a star-star network. Networks 51 (1), 19-33.

Labbé, M., Yaman, H., Gourdin, E., 2005. A branch and cut algorithm for hub location problems with single assignment. Mathematical Programming 102 (2), 371-405.

O'Kelly, M.E., 1986. The location of interacting hub facilities. Transportation Science 20 (2), 92-105. 
O'Kelly, M.E., 1987. A quadratic integer program for the location of interacting hub facilities. European Journal of Operational Research 32 (3), $393-404$. O’Kelly, M.E., Bryan, D., Skorin-Kapov, D., Skorin-Kapov, J., 1996. Hub network design with single and multiple allocation: a computational study. Location Science 4 (3), 125-138.

Pirkul, H., Schilling, D., 1998. An efficient procedure for designing single allocation hub and spoke systems. Management Science 44 (12), S235-S242.

Skorin-Kapov, D., Skorin-Kapov, J., 1994. On tabu search for the solution of interacting hub facilities. European Journal of Operational Research 73 (3), $502-$ 509.

Skorin-Kapov, D., Skorin-Kapov, J., O'Kelly, M.E., 1996. Tight linear programming relaxations of uncapacitated p-hub median problems. European Journal of Operational Research 94 (3), 582-593.

Smith, K., Krishnamoorthy, M., Palaniswami, M., 1996. Neural versus traditional approaches to the location of interacting hub facilities. Location Science 4 (3), 155-171.

Sohn, J., Park, S., 1997. A linear program for the two hub location problem. European Journal of Operational Research 100 (3), $617-622$.

Sohn, J., Park, S., 1998. Efficient solution procedure and reduced size formulations for $p$-hub location problems. European Journal of Operational Research 108 (1), 118-126.

Tan, P.Z., Kara, B.Y., 2007. A hub covering model for cargo delivery systems. Networks 49 (1), 28-39.

Wagner, B., 2004. A note on "The latest arrival hub location problem". Management Science 50 (12), 1751-1752.

Wagner, B., 2008. Model formulations for hub covering problems. Journal of the Operational Research Society 59 (7), 932-938.

Wu, T.H., Kolar, D.J., Cardwell, R.H., 1988. Survivable network architectures for broad-band fiber optic networks: model and performance comparison. Journal of Lightwave Technology 6 (11), 1698-1709.

Yaman, H., 2008. Star p-hub median problem with modular Arc capacities. Computers and Operations Research 35 (9), 3009-3019.

Yaman, H., Kara, B.Y., Tansel, B.C., 2007. The latest arrival hub location problem for cargo delivery systems with stopovers. Transportation Research Part B 41 (8), 906-919. 